PACS numbers: 61.43.Bn, 61.43.Dq, 61.43.Fs, 61.80.Az, 61.82.Bg

\title{
Structure and Radiation Damage of Metallic Glasses
}

\author{
A. S. Bakai \\ National Science Center 'Kharkiv Institute of Physics \& Technology', \\ UA-61108 Kharkiv, Ukraine
}

Two most known models of metallic-glass structure - the model of random closepacked spheres and the polycluster model-are considered. Peculiarities of the structure defects of glasses possessing these structures are compared. Stability, life time, diffusional length of the point defects of the dense random packing and polyclusters are essentially different. As a result, radiation damages of metallic glasses are very sensitive to the structure properties. Description of the relaxation kinetics of irradiated metallic glasses of both structure types is developed. As shown, analyses of the radiation-damage storing and evolution should be an effective tool of the metallic-glass structure investigation.

Розглянуто дві найбільш відомі моделі металевих стекол - модель випадкових щільних упаковок сфер та полікластерна модель. Порівняно особливості структурних дефектів стекол, що мають ту і іншу структуру. Стабільність, час життя, дифузійна довжина точкових дефектів у випадкових щільних упаковках та в полікластерах істотно відрізняються. Через це радіаційні ушкодження металевих стекол виявляються дуже чутливими до властивостей структури. Розвинуто опис релаксаційної кінетики опромінених металевих стекол для обох типів структури. Показано, що аналіз накопичення та еволюції радіаційних пошкоджень може стати ефективним засобом вивчення структури металевого скла.

Рассмотрены две наиболее известные модели металлических стекол модель случайных плотных упаковок сфер и поликластерная модель. Проведено сравнение особенностей структурных дефеектов стекол, имеющих ту или иную структуру. Стабильность, время жизни, диффузионная длина пробега точечных десеектов в случайных плотных упаковках и поликластерах существенно отличаются. Поэтому радиационные повреждения металлических стекол оказываются весьма чувствительными к структурным свойствам. Развито описание релаксационной кинетики облученных металлических стекол для обоих типов структуры. Показано, что анализ процессов накопления и эволюции радиационных повреждений может стать эфффективным средством изучения структуры металлического стекла. 
(Received March 4, 2002)

\section{INTRODUCTION}

Due to remarkable mechanical and physical properties the metallic glasses have many applications. The structure and the properties of these materials are subjects of intensive investigations. The experimental data show that the atomic structure of a metallic glass in many respects is similar to the structure of the supercooled melts. The glass is a nonequilibrium, nearly metastable, state of the matter. It relaxes and crystallises, but the relaxation and crystallization processes are so slow that the term 'frozen-in' is relevant to its structure. The basic property of the metallic glass structure is the topological disorder. Pair correlation of the atom site distribution in the glass promptly disappears with increasing distance and becomes negligible on a distance which is equal to some atom diameters. This property underlies the guesses of a high radiation resistance of the amorphous alloys.

The damage of irradiated crystalline materials starts from displacements of atoms occupying the lattice sites and the subsequent migration of the point defects, getting them at the surface, or recombination on the extended defects (boundaries, dislocations). The annihilation of the interstitial atoms and vacancies also is an effective damage relaxation process. Thus the radiation damage leads to the formation and evolution of crystal defects. Separation of the fluxes of vacancies and interstitials are resulting in the swelling and alloy decomposition processes. The last process is resulting in formation of segregates and change of the phase composition.

The point and extended defects generate configurational disorder. The degree of the configurational disorder increases with the intensity and dose of the irradiation. Some substances (for example, crystalline silicon, alloys $\mathrm{Nb}-\mathrm{Si}, \mathrm{Pd}-\mathrm{Si}, \mathrm{Ni}-\mathrm{Ti}$ and many others) become amorphous under irradiation at rather high radiation doses at low temperatures, close to room one. The process of amorphization starts from accumulation of the structural and compositional disorder in the irradiated crystal. It is naturally to assume, that the irradiation cannot increase the structural disorder of an amorphous substance, which has 'the perfect disorder', and consequently the perfectly disordered amorphous materials should have a high radiation resistance. However this guess, as it will be shown, is not quite correct because of presence of the rather strong short-range order in the metallic glasses. The presence of the local order ensuring stability of the point and extended defects in metallic glasses can significantly affect the kinetics of relaxation of the primary radiation damages and, ultimately, the radiation stability of metallic glasses.

The large sensitivity of the radiation effects to structural features of metallic glasses gives a real opportunity of investigation of their structure 
and structural defects studying the kinetics of accumulation and relaxation of the radiation damages. One of the rather promising methods of investigation of the point defects stability and transport in metallic glasses is the study of processes of the reversible and nonreversible relaxation in samples subjected to irradiation at low temperatures, when transport of vacancies is practically eliminated, but the interstitials can have a noticeable mobility. In this case, the annihilation and recombination on sinks are basic processes limiting accumulation of the point defects.

The flux of interstitials on the extended sinks (their presence in metallic glasses is still under discussion) results in segregation on the sinks and in rearrangements of their microscopic structure. As result of annealing the irradiated sample the vacancies gain mobility and entrapped in the sinks. The vacancies and interstitials have very the same sinks (extended defects) but each sink has different efficiencies for vacancies and interstitials. In the accepted terminology the sinks have some preference for vacancies or for interstitials. The preference is resulting in some separation of the point defect fluxes and, consequently, in the segregation of the ally components and swelling. The point defect relaxation kinetics should be showed up in the recovery stages of electrical resistance. To get more detailed picture of the metal glasses behaviour under irradiation we have to analyse their structural properties and kinetics of the radiation damages.

It is necessary to point out that up to now the microscopic structure of the amorphous solids, and especially of the metal glasses, remains not quite understood because of many difficulties of the direct observation of the disordered structures on the atomic level. Here the kinetics of structural changes of the amorphous metal alloys under irradiation is considered using the most advanced models of the structure. We consider the features of structural transformations under irradiation in alloys possessing the structure of densely random packed spheres (DRPS) [1-4], as well as in alloys possessing the polycluster structure [5,6]. The obtained up to now experimental data allows to prefer the polycluster model of structure of amorphous alloys, though many parameters have to be determined to describe the mechanisms of radiation damage and relaxation kinetics of the amorphous solids As it is shown below the radiation damages and the relaxation kinetics of alloys possessing the DRPS and polycluster structures are essentially different. For this reason investigations of the metallic glass structure evolution under irradiation with subsequent annealing can give important information on the structure properties, point defect stability, diffusion mechanisms and radiation resistance of these materials.

\section{MODELS OF THE METALLIC GLASS STRUCTURE}

Several models of the structure of metallic glasses are proposed, but the 
most advanced and used in interpretation of the physical properties of glasses are the DRPS [1-4] and polycluster [5, 6] models. Let us consider briefly the basic structural properties of the amorphous solids within the framework of these two models.

\subsection{Amorphous Solids of DRPS Structure}

The model DRPS was developed by Bernal [7] to understand the structural properties of fluids. This model and series of its modifications were used also in the physics of metallic glasses due to similarity of the structure properties of fluids and glasses. The pair interaction potential of the ions in metal is characterized by a strong repulsion on the small distances and by a weak attraction on the distances exceeding atomic size. Therefore interaction potential of hard spheres mimics approximately the interaction of the ions in metal. More realistic is the Lennard-Jones potential. The models, in which the Lennard-Jones potential, or another similar potential, is used, are termed as the dense random packing of soft spheres. One more modification of the Bernal model is the random structure consisting of the spheres of different radius. This model was proposed to simulate structure of the amorphous alloys of the metalmetalloid type in which the sizes of atoms of an metalloid are noticeably less than that of the metal atoms. Some structures of the metal-metal type amorphous alloys also were simulated using this model.

A random structure can be covered by the normal tiling using convex polyhedrons. The normal tiling of the structure is termed as that at which the tiling species have common interfaces (polyhedrons have contiguous faces) and there are no parts of the structure which are not covered by the tiling. With Deloney normal tiling in vertexes of the polyhedrons there are atoms, but inside of them there is no atom. These 'empty' polyhedrons are known as Bernal's holes, though as constituent species of the random networks they were introduced long before Bernal [8-10]. A feature of the Bernal hole set is that it includes only five types of the holes: tetrahedrons, octahedrons, trigonal prisms, Archimedean antiprisms and tetragonal dodecahedrons. Concerning the glasses of the metalmetalloid type it is guessed, that the small atoms of the metalloid are disposed in large Bernal's holes formed by the big atoms of the metal [11]. The Bernal's hole containing atom of a metalloid, is termed as a the Bernal-Polk complex.

The problem on the structure defects of amorphous solids has the vital importance. Not going into details (see $[3,5,6]$ ), we have to point out, that the structure evolution and relaxation processes are controlled by transformations and transportation of the structure units which are capable to be changed easy impacted by the thermal fluctuations or external forces. These structure units are responsible for the diffusion transport of atoms, internal friction, plastic and non-elastic strains of the amorphous 
solids. In the crystalline solids the role of such units play the structural defects: point defects (vacancies, interstitials) and extended defects (dislocations, boundaries, interfaces). Atomic structure of the defects in crystals, the modes of their rearrangement under impact of the thermal fluctuations and stresses, their role in kinetic and mechanical processes to the present time are rather well investigated. There is a problem, whether there are similar structure defects in an amorphous solid possessing the DRPS structure or not. A solution of this problem is partially obtained using a numerical simulations of the DRPS [12]. In the simulations a molecular dynamic model was used. With the quenching rate $10^{10}$ $10^{12} \hat{E} / s e c$ it was possible to get an amorphous solid structure. Different pair potentials were used. The defects were generated as following. To generate a vacancy just one of the atoms was taken off. To get the Volterra dislocation the parts of sample were shifted along some cut. The bias vector is the Burgers vector of the dislocation. Annealing of the glassy samples at temperatures below the glass transition temperature, $T_{g}$, was used to investigate stability of the created defects. It has appeared, that the vacancies are unstable: their volume redistributes between nearest atoms, so the vacancies are soon dissolved. Similarly there is a dissolution of the edge dislocation core in the glass. The screw dislocations appear to be something more stable in the DRPS structure than the edge dislocations. These results display, that the point and onedimensional structural defects which are stable in crystals, are not stable in the DRPS structures. Therefore one can not believe that these defects are could be responsible for the structure relaxation and evolution in the DRPS glasses.

In $[13,14]$ the three-dimensional structural defects of the DRPS structures are determined using evaluation of local (in a neighbourhood of each atom) elastic stresses. According to definitions given in $[13,14]$ the depleted zones are identified as the defects of a $n$-type, the zones of high density and high local compression stress are the defects of $p$-type, and regions of high shear strains are the $\tau$-defects. As the local stresses are varying in a wide continuous range of values, a quantitative measure permitting to distinguish the defects and defectless regions is necessary. It is accepted to consider, that the atom belongs to an area of structural defect, if the value of its local stress is deviating from the average value on $10 \%$ or more. Besides the authors has formulated a connectivity condition: not less than $55 \%$ of atoms from its first co-ordination shell should have the same or larger value of the local stress of the same type. Despite of some conventionality of the given definition of the defects it has appeared to be useful in the numerical analysis of the DRPS structures.

Since the point and extended defects are unstable in the DRPS structure, frequently this model is supplemented by the free volume model at attempts to describe kinetic processes, mechanical characteristics and thermodynamics of metallic glasses. Basic in this model is partition of all 
atomic cages on regular (solid-like) and containing free volume (liquidlike) cages. If the volume of an atom cage, $V_{\mathrm{h}}$, exceeds some value, $V_{\mathrm{c}}$, nearly equal to specific volume of the densely packed structure, then the volume $V_{\mathrm{f}}=V_{\mathrm{h}}-V_{\mathrm{c}}$ is termed as free one. Some assumptions on thermodynamics of the free volume exchange between cages and on dependence of $V_{f}$ on the temperature and pressure have to be made to complete the free volume model formulation. In the framework of this model a description of the properties of glasses can be developed. Let us note that the free volume model is compatible to defects of the $n$-type but the $p$ defects and $p-\tau$-defects are beyond of this model.

\subsection{Polycluster Amorphous Structures}

The structure consisting of the set of conjoining locally regular clusters (LRCs) is the polycluster structure. The solid possessing such structure is the polycluster. Figure 1 depicts a fragment of the $2 D$ polycluster. There are the intercluster boundary and the inner boundary are shown on the figure. The polycluster structure is a result of the continuous phase trans-

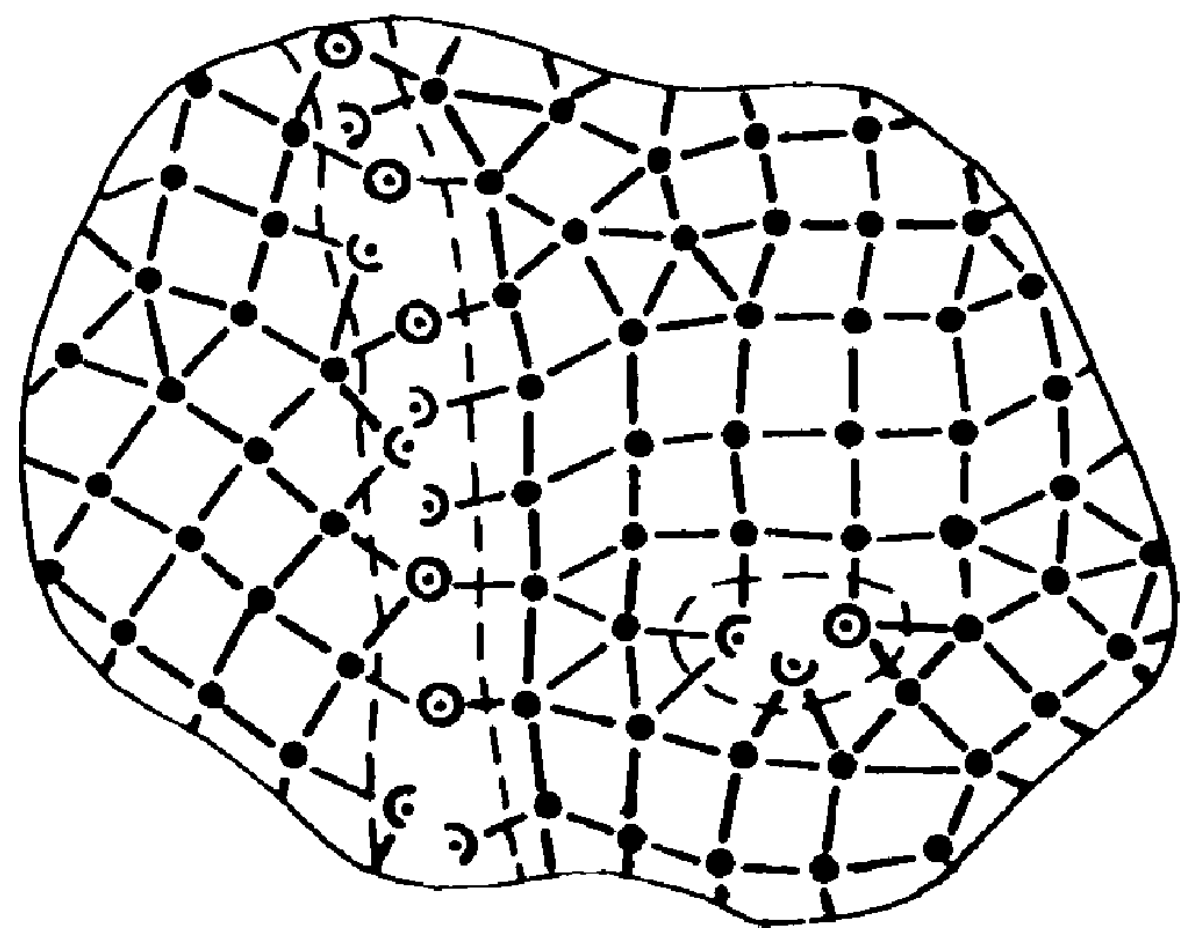

Figure 1. A fragment of the $2 D$ polycluster. Intercluster and inner boundaries are shown. •-regular sites, circles with dot are coincident sites, semicircles with dot are non-coincident sites. 
formation with which the solid-like LRCs growth in the liquid (see [1517]).

The intercluster boundaries have the structure similar to that of the large angle boundaries in polycrystals. The short-range order is essentially disturbed within the boundaries. To get a constructive definition of the cluster boundaries one has to distinguish a perfect (regular) and disturbed short-range order.

The local order of an atom is determined by the configuration of its coordination polyhedron (CP). Between the CP's one may separate sets of topologically equivalent ones. The polyhedrons are topologically equivalent if they have the same number of vertex, edges and faces with identical number of edges meeting in vertexes. We denote by $\left\{\Xi_{\mu}\right\}$ one of such sets, $\mu$ is the index numbering the sets, $\mu=1,2, \ldots, s ; s$ is the number of the local orders, it is an attribute of amorphous solid. Usually in the LRC a few types of the local order are realised.

Let $\left\{E_{\mu}\right\}$ be the set of binding energy values of atoms with topologically equivalent co-ordination of the type $\mu$ and $E_{\mu}^{0}$ is the value of the absolute maximum for this set. Denote by $P_{\mu}^{0}$ the polyhedron on which this maximum is achieved and call it basic. The basic CP is described by a set of vectors (basis) $\left\{\boldsymbol{b}^{0}\right\}$ consisting of the vectors: $\boldsymbol{r}_{i j}^{0}-\boldsymbol{x}_{j}-\boldsymbol{x}_{i} ; i, j==1,2, \ldots, z_{\mu}$, where $x_{i}$ is the co-ordinate of atom inside of $P_{\mu}^{0} ; \tilde{o}_{j}$ are co-ordinates of atoms belonging PCS; $z_{\mu}$ is the co-ordination number.

The CP orientation is determined by three angles, the values of which determine the orientation of the co-ordinate axes system rigidly connected with the polyhedron. Assume that a method is given to choose the co-ordinate system connected with the polyhedron [5] and with this the orientation of each of them is determined.

In the description of the local order, it is important to account for elastic deformations. It is natural to assume that if in a solid the local order is realised with CP from $\left\{P_{\mu}\right\}$, then they differ from the basic polyhedron $P_{\mu}^{0}$ only through elastic deformations. The deformation of polyhedron $P_{\mu}$ is considered to be elastic if the atom binding energy changes monotonously in the process of deformation transferring $P_{\mu}^{\sigma}$ to $P_{\mu}$. The deformation magnitude is described by a set of deformation vectors. Let $\left\{\mathbf{b}_{\mu}\right\}$ be the polyhedron basis of $P_{\mu}$ and $P_{\mu}^{0}$ is the basic polyhedron of the same orientation. Then the set of vectors: $\boldsymbol{u}_{i j}=\boldsymbol{r}_{i j}-\boldsymbol{r}_{i j}^{\circ} \boldsymbol{r}_{i \varphi}, \boldsymbol{r}_{i j} \in\left(\mathbf{b}_{\mu}\right), \boldsymbol{r}_{i j}^{\circ} \in\left(\mathbf{b}_{\mu}{ }^{\circ}\right)$ describes the local deformation.

From the definitions of elastic deformation and the topological equivalence, there follows the definition of the admissible deformation region of co-ordination CPs of each type that we denote by $D_{\mu}$. The boundary of the set of elastic deformations $D_{\mu}$. possesses the obvious property that on it the atom binding energy achieves the minimum.

Note that besides the regular sites and atoms, the LRC contains also irregular ones but such that belong to the CPs of regular sites. The set of irregular atoms forms the LRC boundaries. As is seen, the LRC lattice is 
the locally ordered random network with the intermittent local order and the boundary that is multi-connected in general.

A bounded cluster may be extended by placing atoms on the boundary surface. The completion of boundary site CPs such that these sites become regular is called the regular LRC continuation. The regular continuation of the network is generally ambiguous. The uncertainty of continuation is connected with the admissible variations of elastic deformations of the extended network and also with the possible arbitrariness in the choice of the local ordering of boundary sites, which gives rise to structure frustrations. The ambiguity of the LRC continuation is resulting in the complexity of the glass structure [18].

The regular continuations of LRC networks in the boundary layer play an important role in formation of the boundary structure. Let two LRCs have a common boundary $S_{12}$. Then within the boundary layer the sets of sites of the regular continuations of both clusters are known. Let us denote these sets by $m_{1}$ and $m_{2}$. Two sites, $i \in m_{1}$ and $j \in m_{2}$, are coincident if they may be brought in coincidence by elastic deformation of the regular continuations. If $\rho_{12}$ is the average value of the permissible elastic deformation of the vertexes of co-ordination polyhedrons then the densities of the coincident sites in a boundary layer:

$$
n_{c}^{s}=n^{2} a^{2} \sigma_{12}, \quad \sigma_{12}=\pi \rho_{12}^{2}, \quad n_{u c}^{s}=2 n a\left(1-n a \sigma_{12}\right),
$$

where $n$ is the apparent density the LRC sites; and $a$ is the average interatomic distance. To get these relations it is necessary to mean that the aggregate surface density of sites of regular continuations $m_{1}$ and $m_{2}$ is equal na.

From simple geometry considerations it follows that the mean volume per coincident site is $V_{a}=a^{3}$, while it is about $V_{a} / 2$ for each non-coincident site. Thus, the volume of the hole containing a non-coincident site is essentially larger than the interstitial hole, but is approximately half as large as the vacancy in the LRC body. The arrangement of the atom in the non-coincident site generates comparatively large elastic compression fields. This atom is a partial interstitial $(\tilde{I})$, a vacant non-coincident site represents the partial vacancy $(\widetilde{v})$. Numerical estimates show $[5,6]$ that the formation energy of partial vacancy and partial interstitial are comparable, $E_{\tilde{i}} \approx E_{\tilde{v}} \approx 0.5 \mathrm{eV}$.

The question of atom location in boundary sites requires a special consideration. The non-coincident sites form some connected sets, i.e. the complexes laying in boundary layers. Let us consider the complex consisting of $N$ non-coincident sites with $m$ atoms in them. To find the most probable number of atoms arranged in the complex, we introduce the mean free energy of the complex

$$
F_{N}=E_{N}(m)-S_{N}(m) T .
$$


Here $E_{N}(m)$ is the energy of the complex formation,

$$
E_{N}(m)=m E_{\tilde{i}}+(N-m) E_{\widetilde{v}}-E_{\mathrm{int}}(N, m),
$$

where $E_{\text {int }}$ is the interaction energy of the partial point defects, which is essentially contributed by the interaction through elastic fields.

The entropy $S_{N}(m)$ is contributed by the oscillation and configurational entropies;

$$
S_{N}^{\text {conf }}(m)=k_{\mathrm{B}} \ln \frac{N !}{(N-m) ! m !} .
$$

It was shown [5] that $E_{\text {int }}(N, m)$ has its maximum at $m=N / 2$, when the elastic compression fields generated by the partial interstitials are essentially compensated due to the elastic extension fields surrounding partial vacancies. At $m=N / 2$, the configurational entropy (4) is also maximum. Therefore, $m=N / 2$ is the most probable number of atoms disposed in the complex of $N$ non-coincident sites. In other words, nearly half of the noncoincident sites is occupied by atoms while the another half of the sites is vacant.

One can easy see that the double well potential configurations forming the two-level systems belong to the non-coincident site clusters. Along the non-coincidence boundary sections the high diffusion mobility of atoms takes place. The low-energy structural fluctuations in the polyclusters due to rearrangements of atoms within the non-coincident sections play an important role in low temperature anomalies and the boundary melting process $[5,6]$.

Now the structure defects of the polyclusters can be listed. There are zero-, one-, two-dimensional structural defects exist in the polycluster structures.

Point defects. Vacancies, i.e. vacant regular sites, and the atoms in interstitial cavities, i.e. interstitials, are point defects of LRC. There are no reasons to consider these defects to be unstable. Their stability is provided by the local ordering of the cage forming atoms. Anelastic displacements of surrounding atoms as a result of point defect formation are possible in the places of strong, close-to-critical, elastic distortions of the LRC.

The one-dimensional defects of the polyclusters are the edges of inner boundaries, the triple LRCs junctions and dislocations.

Dislocations. Dislocations in polyclusters exist, but their structure differs from the one in crystals. Because of the lack of translational symmetry, there exist no Burgers vectors that are commensurable with the lattice period even in the LRC body. On the other hand, in a polycluster, one may form the Volterra dislocation cutting along a plane and nonelastically shifting one part of the polycluster relative to the other one in 


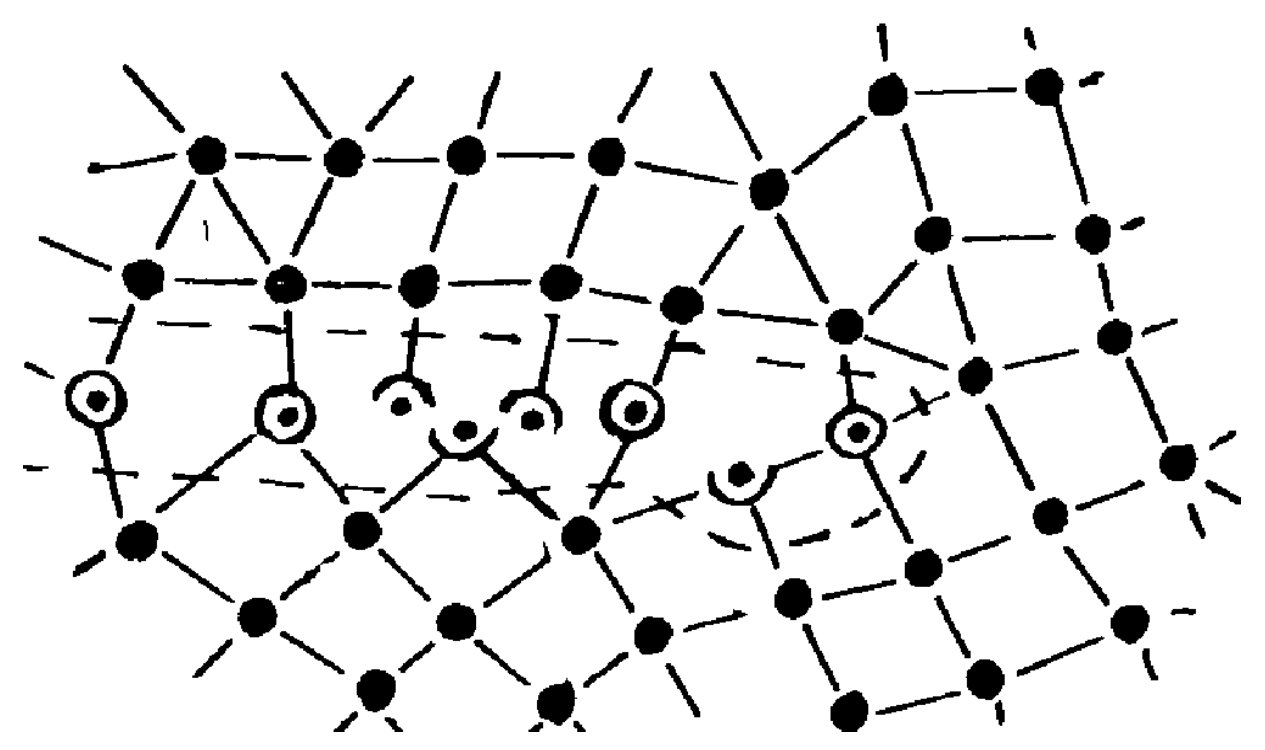

Figure 2. Dislocation structure in LRC: the disordered slip layer and core are shown.

that plane (glide plane). The value of the displacement vector (Burgers vector) must exceed $\rho_{12}$ (and must be equal, nearly $a$ or more) in order to make an inelastic displacement of all sites in the glide layer. The lack of translational invariance and of the natural glide planes in LRC leads to the LO breaking within the glide plane, and it appears to be like to that in the boundary layer. Therefore, the dislocation structure in LRC is such as it is shown in Fig. 2, where the glide plane is locally disordered and its edge is the dislocation core. The field of elastic deformations around the dislocation may be calculated in the continual approximation. Note that a somewhat similar dislocation structure is realised also in some crystals if the Burgers vector does not coincide with the lattice period and in the glide plane the so-called antiphase boundary is formed with the broken LO.

In LRC, one may form prismatic loops by removing the limited plane section and subsequently gluing opposite surfaces. The obtained loop is the edge of the inner boundary layer formed along the glued layers. The field of elastic deformations around such a loop is described in the continual approximation by the same expressions as in crystals. The prismatic loops obtained by locating the additional material in the cut along the layer bounded by the loop have in general similar structure. In clusters as well as in crystals under irradiation, the dislocation loops of vacant and interstitial types may be formed. It is important that the formation of such loops lead to an increase in the density of cluster boundaries.

The surfaces and intercluster boundaries are the most important two- 
dimensional defects.

The three-dimensional or distributed defects, like the defects of $\delta$-, $n$-, and $\tau$-types mentioned above, are not relevant to the polycluster structure.

\subsection{Some Evidences of the Polycluster Metallic Glasses}

Extensive experimental data showing presence of the perfect local order, and the medium range order on distances till 20-30 $\AA$ are reported e.g. in $[3,6]$. Presence of local ordering is the necessary condition of polycluster structures. An important feature of metal glasses is the high density rather large, with volume about $V_{\vec{a}} / 2$, holes. Concentration of these holes can be diminished by the glass annealing at $T<T_{\mathrm{g}}$, the temperature of glass transition [19-21]. In the polycluster amorphous solids these holes are the partial vacancies (vacant non-coincident sites) on the intercluster boundaries. The mentioned experimental data do not contradict as well the model of free volume, but processes which are depending on this free volume (diffusion and a crystallization kinetics) in amorphous alloys, are better compatible with the guess of a polycluster structure of alloys. In [22, 23] it was revealed that the diffusion, which is responsible for the glass crystallization, is controlled by mobile vacancies.

Direct observation of the polycluster structures of the rapidly quenched glassy ribbons as well as bulk metallic glasses was performed using the

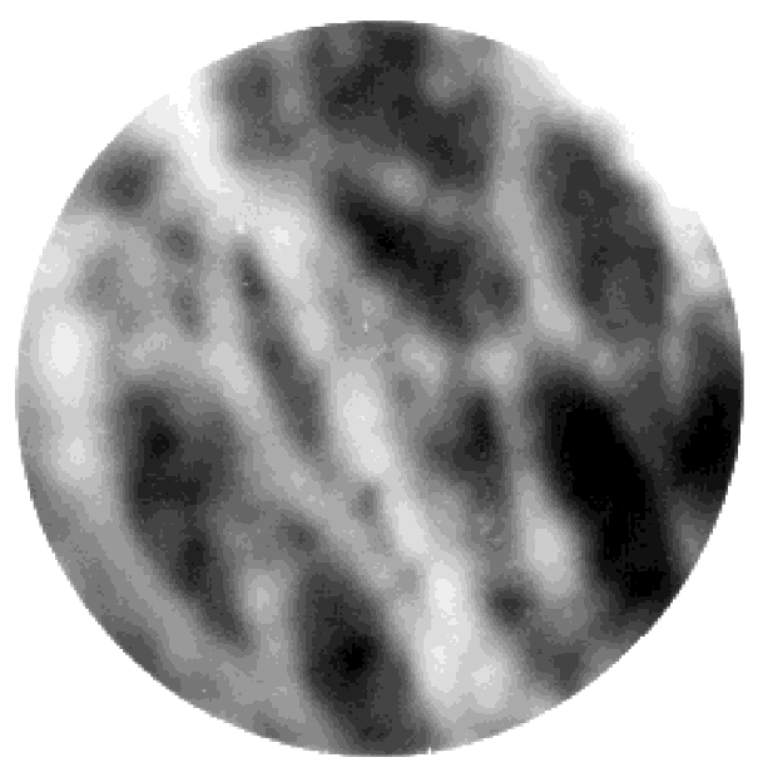

Figure 3. Ion microscopy image of the intercluster boundaries of the bulk metallic glass $\mathrm{Zr}_{41} \mathrm{Ti}_{14} \mathrm{Cu}_{12.5} \mathrm{Ni}_{10} \mathrm{Be}_{22.5}$. The boundaries are decorated by anodic oxidation. 
field emission microscopy [6, 24, 25]. In Figures 3 and 4, results of some recent observations [25] are presented. The surface image of the bulk metallic glass $\mathrm{Zr}_{41} \mathrm{Ti}_{14} \mathrm{Cu}_{12.5} \mathrm{Ni}_{10} \mathrm{Be}_{22.5}$ obtained in the field ion microscope is shown in Fig. 3. The surface anodic oxidation allowed to decorate the intercluster boundaries (bright regions) because the oxidation is more intensive within damaged and defective structure regions. The cluster sizes are varying from 5 to $15 \mathrm{~nm}$.

In Figure 4a, the autoelectronic image of the pin tip of the same glass

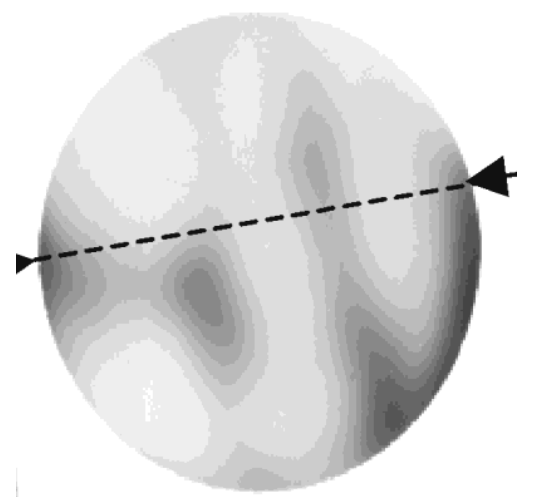

a

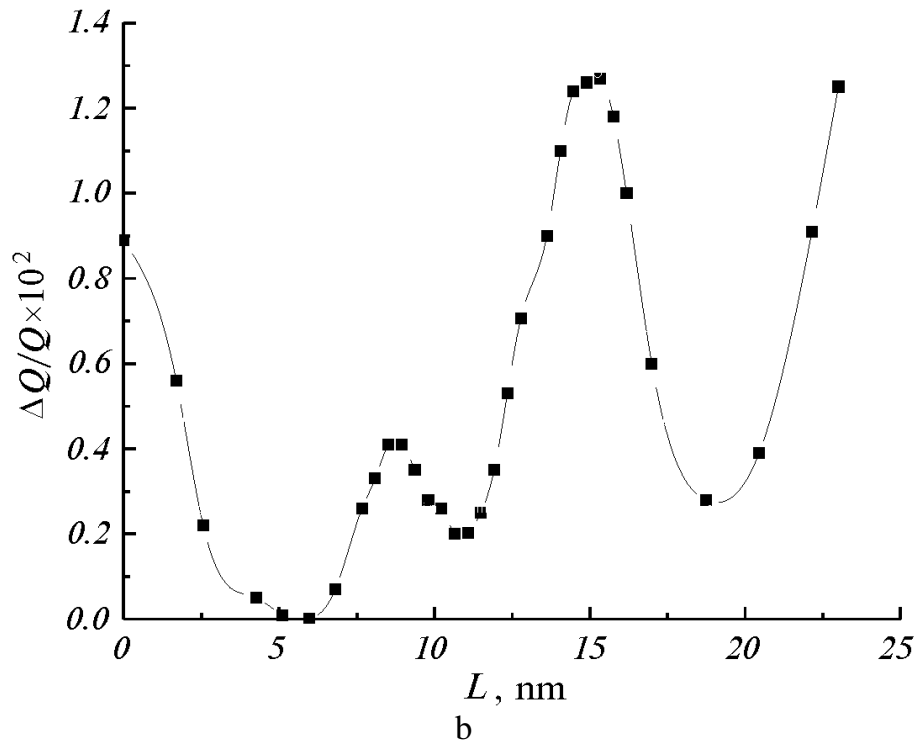

Figure 4. The autoelectronic evaporation image of the $\mathrm{Zr}_{41} \mathrm{Ti}_{14} \mathrm{Cu}_{12.5} \mathrm{Ni}_{10} \mathrm{Be}_{22.5}$ bulk metallic glass (a) and the evaporation field energy along the shown crosssection (b). 
is shown. The brightness is proportional to the energy of the field evaporation. The variation of the field evaporation energy, $\Delta Q / Q$, along the cross-section, marked by the dashed line in Fig. $4 a$, is shown in Fig. $4 b$. Here $Q$ is the field evaporation energy, $\Delta Q$ is its variation.

It is seen that the width of the intercluster boundary is nearly $1 \mathrm{~nm}$, while the binding energy of atoms within the boundaries is on 0.13$0.43 \mathrm{eV}$ less than in the cluster bulk.

In spite of the eloquent evidences of the polycluster structure of some metallic glasses the problem of the structure properties and defects of metallic glasses is not exhausted. The relaxation kinetics, plastic deformations, hardness, compositional stability and crystallization processes-all these processes are sensitive to the peculiarities of the structure which have to be investigated and established.

\section{ELEMENTARY RADIATION DAMAGES OF AMORPHOUS SOLIDS}

Independently of the solid body structure the atomic radiation damage starts from the primary knocked-on atom (PCA) generation by an irradiation. The stage of collisions of the PCA up to the moment when inelastic displacements of atoms, as a result of collisions with the PCA, is called the dynamic stage. The characteristic time of the dynamic stage does not exceed about $10^{-12} \mathrm{sec}$. Afterward there occurs the kinetic structural relaxation of the radiation damage starts.

Peculiarities of the structure of amorphous solids, polyclusters and DRPS structures, are resulting in rather considerable difference of the both dynamic and kinetic stages of the radiation damage and relaxation within them.

Let us consider in the first instance the DRPS structure. The PCA formation accompanies within them, like in crystals, by inelastic displacements of environmental atoms, if the energy of the PCA is big enough. It is important to know, which kind of the structure defects can be formed on the dynamic stage.

The stable Frenkel pairs in the DRPS structure can not be formed because the vacancies, as well as interstitials, are unstable within it [12]. The three-dimensional, distributed defects of the $\delta$ - and $n$-types can be formed on the late dynamic stage as a result of the Frenkel pair 'dissolution'. Thus unlike to the crystalline solids, in the DRPS structure the defect formation can not have the sharp energy threshold of the atom displacement, and inelastic rearrangements are resulting in accumulation of the elastic deformation energy and enhancing of the density fluctuation. No stable point defects or their aggregation can be formed under irradiation.

The displacement cascades within the DRPS structure can be formed if the PCA has big enough energy but the structure of cascade region is completely different as compare to that in crystals. The number of atoms 
displaced within the cascade can be rather large but one has not to expect that a depletion zone surrounded by 'cloud' of interstitials can be formed because formation of the crowd ions and focused collision chains is impossible in a randomly packed structure.

The process of defect generation by irradiation in the polyclusters is very similar to that in crystals. Irradiation generates Frenkel pairs within LRCs and the threshold energy is comparable with that in crystals. Of course, there is a distribution of the threshold energies in polyclusters due to the structure randomness, but the distribution variance cannot be large because it has to be like the variance of the atom binding energy which is estimated to be less than $1 \mathrm{eV}$ (see Fig. 4). The cascade structure in polyclusters differs from that in crystals. First of all it is because the length of focused collision chains is shorter in the non-crystalline structures. Secondly, as it was revealed, the LRCs have the characteristic size $\sim 10 \mathrm{~nm}$. Therefore the displacement cascades with typical sizes of a few $\mathrm{nm}$ are either close to intercluster boundaries or overlap them. The boundaries are sinks of the point defects. As it is shown in [22] the vacancies and interstitials are annihilating in the non-coincident sites as following

$$
\tilde{v}+i=\tilde{i}, \tilde{i}+v=\tilde{v},
$$

where $v$ and $i$ denote vacancies and interstitials respectively; the partial vacancies and interstitials are denoted by $\tilde{v}$ and $\tilde{i}$.

The density of the irradiation generated defects within cascade region on the post-dynamic stage is less in the polyclusters than in crystals due to the mentioned above reasons.

In $[26,27]$ the threshold energy of the defect generation in amorphous $\mathrm{Fe}-\mathrm{B}$ and $\mathrm{Pd}-\mathrm{Si}$ under electron irradiation was measured. It turns out that they coincide with the Frenkel pair threshold energy in crystalline alloys. These results show that the elementary processes of the point defect generation under irradiation in metallic glasses are very similar to that in crystals. One can conclude that the local order in the metallic glasses is rather perfect. This evidence supports the relevance of the polycluster model to the amorphous alloys.

In Refs. [20, 22, 23], it is reported that generation of the vacancy-like defects of the volume about $v_{\mathrm{a}}$ which are recombining due to the annealing in a wide temperature range. Along with that the concentration of smaller voids, which are existing in the alloys even without irradiation, does not change considerably during irradiation or annealing stages. This evidence also supports the polycluster model of the amorphous alloys. The small voids in the polyclusters are identified as the partial vacancies within boundaries. Because the boundaries are stable defects, the concentration of the partial vacancies attributed to the boundaries, can not change considerably due to irradiation or annealing. Along with that the regular vacancies within the LRC body are mobile in a proper tempera- 
ture range and can be absorbed on boundaries (3).

\subsection{The Kinetic Relaxation Stage}

Relaxation of the irradiation generated defects in the polyclusters and DRPS structure on the kinetic stage is rather different. In the DRPS structure the kinetic of comparatively large density fluctuations ( $p$ - and $n$ defects) can be described by the diffusion equation describing evolution of the density $n(\boldsymbol{x}, t)$

$$
\frac{\partial \tilde{n}(\mathbf{x}, t)}{\partial t}-\frac{\partial}{\partial \mathbf{x}} D \frac{\partial}{\partial \mathbf{x}} \tilde{n}(\mathbf{x}, t)=g_{r}(\mathbf{x}, t)
$$

where $\tilde{n}(\boldsymbol{x}, t)=n(\boldsymbol{x}, t)-\bar{n}$ describes the density fluctuations, $\bar{n}$ is the average density. The function $g_{r}(\boldsymbol{x}, t)$ describes formation of the density fluctuations under irradiation. The diffusion coefficient accordingly to the free volume model [28] is

$$
D=(1 / 6) v a^{2} \exp \left(-V^{*} / V_{\mathrm{f}}\right),
$$

where $v$ is the frequency of atom oscillations, $V^{*}$ is about $v_{\mathrm{a}}$, and $V_{\mathrm{f}}$ has to be substituted by the local value of the free volume, $V_{f}(\boldsymbol{x}, t)=n^{-1}(\boldsymbol{x}, t)$ $-v_{\mathrm{a}}=(\bar{n}+\widetilde{n})^{-1}-\boldsymbol{V}_{\mathrm{a}}$. This quantity, unlike $V_{\mathrm{f}}$, is negative within regions where $n^{-1}(\boldsymbol{x}, t)<v_{\mathrm{a}}$. There is no diffusion in these regions.

The density fluctuations formation starts from generation of vacancies and interstitials which afterward transform in $n$ - or $p$-defects is consisting of 10-20 atoms, the characteristic liner size of these defects is a few a. Therefore the spectral density of $g_{r}(\boldsymbol{x}, t)$ has a maximum at small, comparable to $a$, wave lengths. Besides $g_{r}(\boldsymbol{x}, t)$ is proportional to the frequency of the PCA generation.

The most important peculiarity of the relaxation process described by Eq. (6) is that it does not connected with a long-range transport in contrast to the point defect relaxation in crystals where the characteristic defect to sinks. In order words, in the DRPS structure the point defect sinks are distributed everywhere.

It has to be concluded that an irradiation can generate density fluctuations in the DRPS structure. It changes also the diffusion rate. But in total these structures are radiation resistant.

The structure relaxation kinetics in the polyclusters is very similar to that in polycrystals consisting of small grains. The boundaries in both polyclusters and polycrystals are the dominating sinks of the point defects.

Each type of the local order (type of the co-ordination polyhedron $P_{\mu}$ ) is characterized by the energy of vacancy formation $E_{\mathrm{v} \mu}$. In analogy the interstitial formation energy, $E_{\mathrm{i}}$, depends on the environment of the interatomic cavities (they are numbered by the index ' $v$ '). Along with 'normal' 
interstitials in LRCs the interstitials having double configuration evidently can exist because the short-range order is responsible for the configuration and stability of the point defects while the long-range order nothing to do with that.

The point defect kinetics can be described by equations similar to that used for crystalline solids

$$
\frac{\partial C_{i, v}}{\partial t}+\operatorname{div} j_{i, v}=K-g_{i v} C_{i} C_{v}, j_{i, v}=-D_{i, v} \nabla C_{i, v},
$$

where $C_{\mathrm{i}, v}$ are concentrations of interstitials and vacancies, $K$ is the generation rate of point defects, $g_{\mathrm{iv}}$ is the recombination rate and $D_{\mathrm{i}, \mathrm{v}}$ are the diffusion coefficients.

The equations (7) has to be accompanied by the equations determining the partial concentrations of vacancies (or interstitials) of different types:

$$
C_{v}=\sum_{\mu} C_{v, \mu} ; \quad C_{v, \mu} / C_{v, v}=\exp \left[\left(E_{v, v}-E_{v, \mu}\right) / k T\right] .
$$

Here $C_{v, \mu}$ is the partial concentration of vacancies (they are in sites with $\mu$-th local order).

A similar to (8) equation is valid for interstitials too. Arrived to boundaries point defects a recombining accordingly to the scheme (5). It is seen that the partial vacancy is transforming into a partial interstitial after absorption of the arrived interstitial. This last one is transforming into a partial vacancy in result of the vacancy absorption. Thus non-coincident sites are non-saturating and non-preferential sinks of the point defects. They just alternate their ability to absorb vacancy or interstitial in accord with (5). Due to this exceptional property the non-coincident sites are ideal sinks of the point defects. Due to the high recombination rate of the point defects the swelling of the polyclusters to be damped [29]. A similar properties have also finegrained polycrystals [30]. It is revealed experimentally that the fine-grained materials (the grain size was about $1 \mu \mathrm{m}$ ) do not swell and that in the vicinity of surface the materials are free of voids [31]. In [32] the thickness of the free of voids surface layer of nickel was measured to be $10^{2} \mathrm{~nm}$ at $T=330^{\circ} \mathrm{C}$.

As a low temperature, when vacancies are almost immobile, the point defect recombination in the LRC body is the main mechanism of their losses. This process is described by the second term of r.h.s. (7).

If just the interstitials are mobile, they occupy more and more partial vacancies on boundaries. By this way the segregation of the most mobile interstitials in boundaries takes place. As a result the two-dimensional structure defects, similar to the Guinier-Preston zones, are forming within boundaries. 


\subsection{Mechanisms of the Irradiation Impact on the Kinetics of Struc- ture Evolution in Amorphous Alloys}

While in the DRPS structure the radiation stimulation of the diffusion due to generation of the heterogeneities is not important, in the polyclusters the radiation stimulated diffusion enhance the effects controlled by the long-range transport. The concentrations of the vacancies and interstitials in the irradiated polycluster can be estimated using the stationary solution of $(7)$

$$
C_{\mathrm{v}, \mathrm{i}}=r_{\mathrm{LRC}}^{2} K / D_{\mathrm{v}, \mathrm{i}}
$$

Here $r_{\mathrm{LRC}}$ is the average size of LRC. Because this quantity is small $(\sim 10 \mathrm{~nm})$ the consecrations of the point defects can be considerably large at the temperature range where their diffusion mobility is small.

Let us consider a two-component alloy consisting of atoms of $A$ and $B$ types. The diffusion coefficient of $A$ atoms, as it follows from the expression (9), is

$$
D_{A}=C_{v} d_{v, A}=\frac{K r_{L R C}^{2} d_{v, A}}{C_{A} d_{v, A}+C_{B} d_{v, B}},
$$

where $\tilde{N}_{A}, \tilde{N}_{B}$ are the concentrations of atoms $A$ and $B, d_{v A}, d_{v B}$ are their partial diffusion coefficients by the vacancy mechanism. The expression (10) is obtained in the guess, that the vacancy concentration generated under the irradiation, is much larger than their equilibrium concentration.

The experimental observations of the radiation enhanced diffusion in the amorphous alloys $\mathrm{Fe}-\mathrm{Ni}-\mathrm{E}-\hat{\mathrm{A}}$ and $\mathrm{Ni}-\mathrm{Nb}$ are reported in $[22,23]$. The vacancy mechanism of the diffusion was identified and the concentration of sinks of the point defects in these alloys are estimated to be $\sim 10^{-2}$ per atom. It is in accord with obtained in $[5,6]$ estimations of the LRC sizes $(\sim 10 \mathrm{~nm})$ : the concentration of partial point defects in the polyclusters is $\sim 10^{-2}$.

A change of the crystallization rate of the amorphous alloys under irradiation was observed in the series of experiments [22, 23, 33, 34]. In Ref. [33] it is revealed, that the crystallization of the amorphous alloy Àu-Si under electron irradiation happens at the temperature which is much lower than the temperature of crystallization of the non-irradiated alloy. In Ref. [34] the temperature of crystallization of the amorphous alloy Fe-B has decreased due to the low-temperature irradiation by neutrons. Along with that, the crystallization temperature of the amorphous alloy Fe-Â- $\bigoplus$ has increased after the same irradiation.

Impact of the irradiation on the crystallization of polycluster amorphous alloys happens not only due to the diffusion enhancement but also owing to segregation of the alloy components due to the difference of the partial diffusion coefficients, $d_{i A}, d_{v A}$ and $d_{i B}, d_{v B}$, the same as in crystals $[35,36]$. Gra- 
dient of the atom A concentration in the vicinity of the cluster boundary can be estimated using the following expression [36]:

$$
\left.\begin{array}{l}
\nabla C_{A}=\frac{C_{A} C_{S}\left(d_{v, A} d_{i, B}-d_{i, A} d_{v, B}\right)}{C_{S} d_{i S} D_{A}+C_{A} d_{i A} D_{B}} \nabla C_{i} \\
D_{A}=C_{v} d_{v A}+C_{i} d_{i A}, D_{B}=C_{v} d_{v B}+C_{i} d_{i B}
\end{array}\right\}
$$

Formation of the inhomogeneities of concentrations under irradiation is possible also in the LRC body like it was observed in the crystalline alloys [37]. A nonuniform distribution of the point defects in LRC, as it is seen from (8), is stipulated by inhomogeneity of the local order. The redistribution of concentration of the alloy components can be accompanied by changes of local ordering of atoms, that in turn, changes quasi-stationary concentrations of the point defects according to the expression (8).

In the DRPS structures because of lack of the extended onedimensional and two-dimensional sinks (and also because of instability of the point defects) the segregation of alloy components on sinks is impossible. Therefore in the metallic glasses possessing DPRS structure the segregation under irradiation is not relevant.

\section{CONCLUDING REMARKS}

Stability, life time, diffusion length of the point defects of the dense random packing and polyclusters are essentially different. As result, the radiation damages of metallic glasses is very sensitive to the structure properties. We have considered the relaxation kinetics of irradiated metallic glasses of both structure types. Apparently the both considered structure models have to be treated as idealised ones. Nevertheless it is believed that they properly reflect the main properties of some types of the amorphous metals and alloys. The polycluster structures are revealed by the field emission microscopy investigations of many metallic glasses while the DRPS model supposedly is relevant to the amorphous metallic films obtained by the atom flux deposition on cryogenic substrates. It is seen that analysis of the radiation damage storing and evolution should be an effective tool of the metallic glass structure investigation.

A promising investigation procedure is to irradiate an amorphous metal or alloy at low temperature when the point defects (if they are stable) are admittedly immobile. Nevertheless the processes of the vacancyinterstitial annihilation due to the short-range attraction cannot be damped at any temperature because the Frenkel pairs are unstable since the distance between vacancy and nearest interstitial is less then annihilation radius which is equal to some interatomic distances. The consequent annealing procedures should lead to activation of another relaxation processes controlled by the point defect diffusion. Due to the glass 
heterogeneities the relaxation activated processes are not homogeneous and have a wide range of the activation energies and relaxation times. Nevertheless likely it is possible to resolve the relaxation stages connected with the mobility activation of different defects and their complexes. This approach was very fruitful for investigations of the relaxation kinetics of crystalline materials so it is expected to be useful also for the non-crystalline solids but in this last case the analysis procedure should be more sophisticated. Some attempts in this direction were undertaken (e.g. [38]) but more systematic investigations are desirable.

\section{ACKNOWLEDGEMENT}

This work was made at financial support of STCU, Project No. 655.

\section{REFERENCES}

1. À. İ. l̀ànokhin, B. S. Mitin, and V. A. Vasilev, Amorphous Alloys (Moscow: Metallurgiya: 1984) (in Russian).

2. Topics in Applied Physics, vol. 46: Glassy Metals I (Eds. H.-J. Guentherodt and H. Beck) (Heidelberg-Berlin: Springer-Verlag: 1981).

3. Topics in Applied Physics, vol. 53: Glassy Metals I (Eds. H.-J. Guentherodt and H. Beck) (Heidelberg-Berlin: Springer-Verlag: 1983).

4. K. Suzuki, H. Fujimori, and K. Hashimotyo, Amorphous Metals (Moscow: Metallurgiya: 1987) (Russian translation).

5. A. S. Bakai, Polycluster Amorphous Solids (Moscow: Ehnergoatomizdat: 1987) (in Russian).

6. A. S. Bakai, Applied Physics, vol. 72: Glassy Metals III (Eds. H. Beck and H.-J. Guentherodt) (Heidelberg-Berlin: Springer-Verlag: 1994), p. 208.

7. J. Bernal, Proc. Roy. Soc. A, 280: 299 (1964).

8. J. F. Voronoy and J. Reine, Angew. Math., 134: 198 (1908).

9. J. F. Voronoy, Collected Paperss (Kiev: Naukova Dumka: 1952), vol. 2 (in Russian).

10. B. N. Delone, Uspekhi Mat. Nauk, Iss. 3: 3 (1937) (in Russian).

11. D. Polk, Acta Met., 20: 485 (1972).

12. P. Chaudkhary, S. Spaepen, and P. J. Steinhardt, Topics in Applied Physics, vol. 53: Glassy Metals I (Eds. H.-J. Guentherodt and H. Beck) (Heidelberg-Berlin: Springer-Verlag: 1983), chap. 5.

13. T. Egami, K. Maeda, and V. Vitek, Phil. Mag. A, 41: 883 (1980).

14. D. Srolovitz, K. Maeda, V. Vitek et al., Phil. Mag. A, 44: 847 (1981).

15. A. S. Bakai, Low Temp. Phys., 22, No. 8: 733 (1996); ibid., 24, No. $1: 3$ (1998).

16. A. S. Bakai, Cond. Mat. Phys., 3: 675 (2000).

17. A. S. Bakai, Ukr. J. Phys., 45: 508 (2000).

18. R. G. Palmer, Adv. Phys., 31: 669 (1982).

19. E. Cartier, F. Heinrich, and H. Guentherodt, Phys. Lett. A, 81: 393 (1981).

20. P. Moser, P. Hautojarvi, and I. Ili-Kaupila, Rad. Eff., 62: 153 (1982).

21. A. Audouard, P. Moser, and P. Hautojarvi, Rad. Eff., 70: 285 (1983).

22. Y. Limoge, Z. Phys. Chem. Neue Folge, 156: 391 (1988). 
23. Y. Limoge, J. Non-Cryst. Solids, 117\&118: 708 (1990).

24. A. S. Bakai, V. V. Kul'ko, I. M. Mikhailovskij, V. B. Rabukin, and O. A. Velikodnaya, J. Non-Cryst. Solids, 182: 135 (1995).

25. A. S. Bakai, I. M. Mikhailovskij, T. I. Mazilova, and N. Wanderka, Low Temp. Phys., 27, No. 4: (2002).

26. A. Audouard, J. Balogh, I. Dural, and J. C. Jonset, Rad. Eff., 70: 285 (1983).

27. S. Klaumumer and G. Schumacher, Acta Met., 30: 1493 (1982).

28. F. Spaepen, Acta. Met., 25: 407 (1976).

29. A. S. Bakai, Pis'ma ZhTF, 9: 1477 (1983) (in Russian).

30. V. F. Zelenskij et al., Some Problems of Radiation Damage Physics (Kiev: Naukova Dumka: 1979) (in Russian).

31. Yu. V. Konobeev et al., Phys. Stat. Sol. A, 29: K121 (1975).

32. D. Norris, Rad. Eff., 15: 1 (1972).

33. İ. Kiritani, Vopr. At. Nauki Tekhn. Ser.: PRDRM, Iss. 1(29)-2(30): 74 (1984).

34. I. A. Naskidashvili et al., Vopr. At. Nauki Tekhn. Ser.: PRDRM, Iss. 1(39): 30 (1987).

35. A. D. Marwick, J. Phys. F, 8: 1849 (1978).

36. H. Wiedersich, P. Okamoto, and N. Lam, J. Nucl. Mater., 83: 98 (1979).

37. G. Martin, Phys. Rev. B, 21: 2122 (1980).

38. G. Schumacher, S. Klaumuenzer, W. Petry, G. Wallner, W. Weck, and U. Dedek, Z. Phys. Chemie Neue Folge, 157: 313 (1988). 